\title{
Compton sources for the observation of elastic photon-photon scattering events
}

\author{
D. Micieli, ${ }^{1}$ I. Drebot, ${ }^{2}$ A. Bacci, ${ }^{2}$ E. Milotti, ${ }^{3}$ V. Petrillo, ${ }^{2,4,}{ }^{*}$ M. Rossetti Conti, ${ }^{2,4}$ \\ A. R. Rossi, ${ }^{2}$ E. Tassi, ${ }^{1}$ and L. Serafini ${ }^{2}$ \\ ${ }^{1}$ Universitá degli Studi della Calabria, via Pietro Bucci, 87036 Arcavacata di Rende (Cosenza) Italy \\ ${ }^{2}$ INFN-Sezione di Milano, via Celoria 16, 20133 Milano, Italy \\ ${ }^{3}$ Universitá di Trieste and INFN-Sezione di Trieste, Via Valerio 2, 34127 Trieste, Italy \\ ${ }^{4}$ Universitá degli Studi di Milano, via Celoria 16, 20133 Milano, Italy
}

(Received 13 May 2016; published 1 September 2016)

\begin{abstract}
We present the design of a photon-photon collider based on conventional Compton gamma sources for the observation of elastic $\gamma \gamma$ scattering. Two symmetric electron beams, generated by photocathodes and accelerated in linacs, produce two primary gamma rays through Compton backscattering with two high energy lasers. The elastic photon-photon scattering is analyzed by start-to-end simulations from the photocathodes to the detector. A new Monte Carlo code has been developed ad hoc for the counting of the QED events. Realistic numbers of the secondary gamma yield, obtained by using the parameters of existing or approved Compton devices, a discussion of the feasibility of the experiment and of the nature of the background are presented.
\end{abstract}

DOI: 10.1103/PhysRevAccelBeams.19.093401

\section{INTRODUCTION}

The scattering of light by light $(\gamma \gamma \rightarrow \gamma \gamma)$, a phenomenon which is precluded in classical electrodynamics in vacuo, is one of the most elusive effects foreseen by quantum electrodynamics (QED). Immediately after the appearance of the Dirac's theory of the electrons, it became clear that the existence of antiparticles opened the way to $\gamma \gamma$ scattering [1], that has never been observed so far, except as radiative correction to other processes. Apart from some early misdirected or technologically immature attempts, the first experimental proposals, dated back to the 1960s, all involved the use of low-energy photons, with energies that were mostly not higher than a few $\mathrm{keV}$ [2-4]. Low-energy photon-photon scattering can be described by the effective Euler-Heisenberg-Weisskopf Lagrangian [5], which allows the calculation of the propagation properties of light in vacuum, either in the presence of other real photons, or within the sea of virtual photons produced by external static fields. The measurement of photon-field scattering appears to be advantageous with high-field magnets, and, in this case, the phenomenon manifests itself as an effective birefringence of vacuum produced by the magnetic field [6-9]. The forthcoming availability of extremely high intensity laser sources has also stimulated many experimental proposals aimed to observe the scattering of near-

\footnotetext{
*Corresponding author.

Petrillo@mi.infn.it

Published by the American Physical Society under the terms of the Creative Commons Attribution 3.0 License. Further distribution of this work must maintain attribution to the author $(s)$ and the published article's title, journal citation, and DOI.
}

visible photons of high-intensity beams [10,11]. The experiments that utilize near-visible photons present the problem that the photon-photon cross section is extremely low at energies of a few $\mathrm{eV}$, and the induced birefringence has yet to be detected $[12,13]$. On the other hand, the unpolarized cross section grows as the sixth power of the center of mass $(\mathrm{CM})$ energy and reaches a peak value of about $1.6 \mu \mathrm{b}$ at a CM energy around $1.6 \mathrm{MeV}$. Experiments in this energy range can be an appealing alternative [14-20], encouraged also by the development of highbrilliance photon sources in the range of hard x-ray and gamma wavelengths [21], which can permit indeed to reach and explore the region around the cross section peak.

The purpose of this paper is to present the design of a $\gamma \gamma$ collider based on conventional Compton gamma sources. Two symmetric electron beams, generated by photocathodes and accelerated in linacs, produce two primary gamma rays through Compton backscattering with two high energy lasers. The two primary gamma pulses cross and scatter. Tuning the gamma energies to the energy corresponding to the maximum of the photon-photon cross section, a significant amount of secondary gamma photons is generated. Figure 1 presents the scheme of the source.

The whole process is analyzed by start-to-end simulations. The electron beams are tracked with the code ASTRA [22], while the primary gamma photons have been produced by means of CAIN [23]. A new Monte Carlo code named ROSE (Rate of Scattering Events) has been developed ad hoc for the counting of the QED events. Realistic numbers of secondary gamma yield, obtained with the parameters of commissioned or approved Compton sources, together with a discussion of the feasibility of 


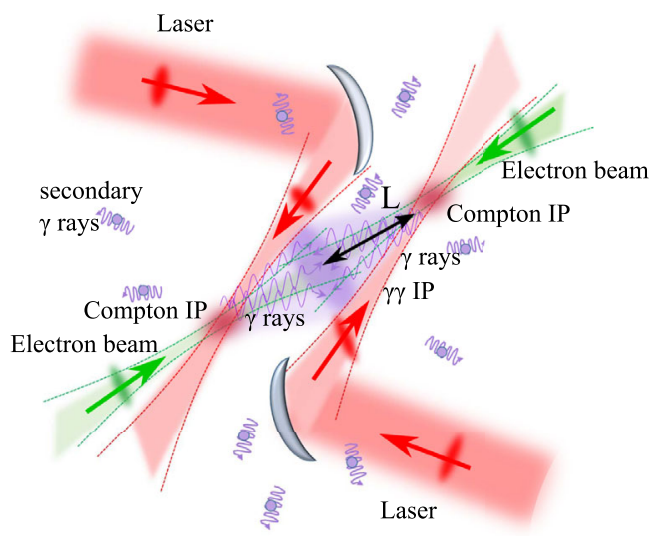

FIG. 1. Scheme of the $\gamma \gamma$ interaction. Two lasers (in red) impinge on two electron beams (in green) in two interaction points (Compton IP), generating primary gamma rays (in violet). The primary gamma rays interact in the $\gamma \gamma$ IP, generating secondary gammas.

the experiment, are presented. Finally, we comment on the opportunity of studying the Breit-Wheeler [24] process, still unobserved to this date.

\section{II. $\gamma \gamma$ KINEMATICS AND CROSS SECTION}

The kinematics of the head-on $\gamma \gamma$ reactions predicts that, assigned the invariant mass $\sqrt{s}=\sqrt{2 E_{1} E_{2}\left(1-\underline{e}_{1} \cdot \underline{e}_{2}\right)}$ $\left(E_{1}, E_{2}\right.$ being the energies of two primary photons with momenta directed respectively along $\underline{e}_{1}$ and $\underline{e}_{2}$ in the laboratory) and assuming $\underline{e}_{1}=-\underline{e}_{2}$, the photons acquire respectively the energies

$$
\begin{gathered}
E_{3}=\frac{2 E_{1} E_{2}}{E_{1}+E_{2}+\left(E_{1}-E_{2}\right) \cos \theta_{3}} \\
E_{4}=E_{1}+E_{2}-E_{3}
\end{gathered}
$$

whereas the momentum angles $\theta_{3}$ and $\theta_{4}$ satisfy

$$
E_{3} \sin \theta_{3}=E_{4} \sin \theta_{4} \text {. }
$$

Figure 2 shows the energy-angle correlation in the laboratory frame. For the symmetric head-on collisions $\left(E_{1}=E_{2}\right)$, described in (a), the two emitted photons have equal energies and are anticollinear. For asymmetric scattering (b) and (c), instead, the most energetic particle is always boosted forwards, while the least energetic can be comoving or going backwards. A geometrical separation between primary and secondary $\gamma$ photons can be advantageous for their detectability. In this figure, therefore, the regions where $\pi / 2<\theta_{3,4}<3 \pi / 4$ are highlighted.

Figure 3 shows the elastic cross section of the $\gamma \gamma$ scattering for unpolarized and polarized photons as a function of the CM energy $\sqrt{s}$, as calculated from the amplitudes in Refs. [25-27].

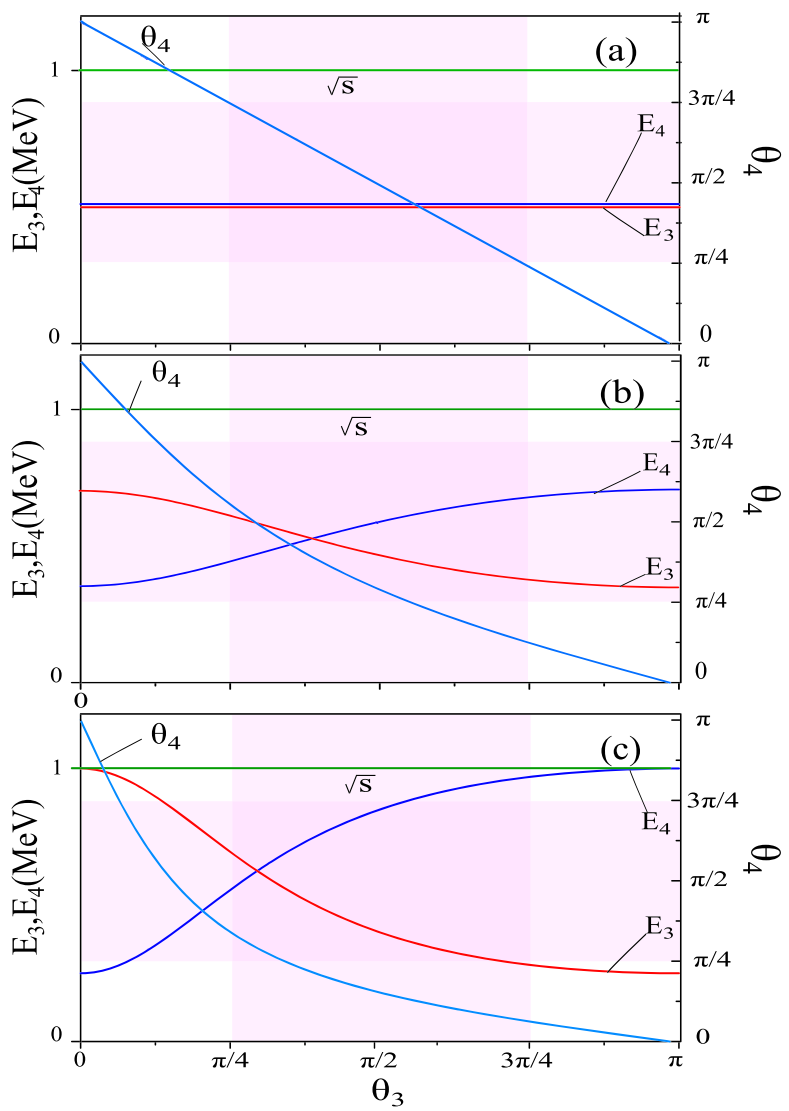

FIG. 2. Energies $E_{3}$ and $E_{4}$ and angle $\theta_{4}$ as a function $\theta_{3}$ for: (a) $E_{1}=E_{2}=0.5 \mathrm{MeV}$, (b) $E_{1}=0.707 \mathrm{MeV}$ and $E_{2}=0.353 \mathrm{MeV}$, and (c) $E_{1}=1 \mathrm{MeV}$ and $E_{2}=0.25 \mathrm{MeV}$.

Taking into account the linear polarization of the primary photons ( 1 is the linear polarization perpendicular to the scattering plane, 2 is the linear polarization parallel to the scattering plane) and averaging over the final polarization state $\left(\lambda_{3}\right.$ and $\left.\lambda_{4}\right)$, Fig. 3 shows that the symmetric scattering with same photon polarization $(\sigma \approx 0.83 \mu \mathrm{b}$ for polarization 1 or $\sigma \approx 1.19 \mu \mathrm{b}$ for polarization 2) has a lower probability with respect to the case with crossed polarizations $(\sigma \approx 2.41 \mu \mathrm{b})$. For symmetric collisions, where the

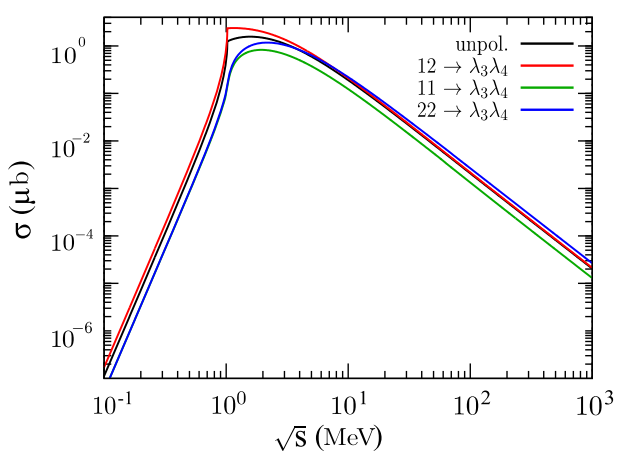

FIG. 3. Semipolarized (linear polarization) and unpolarized total cross section of $\gamma \gamma$ scattering as a function of center of mass energy. 


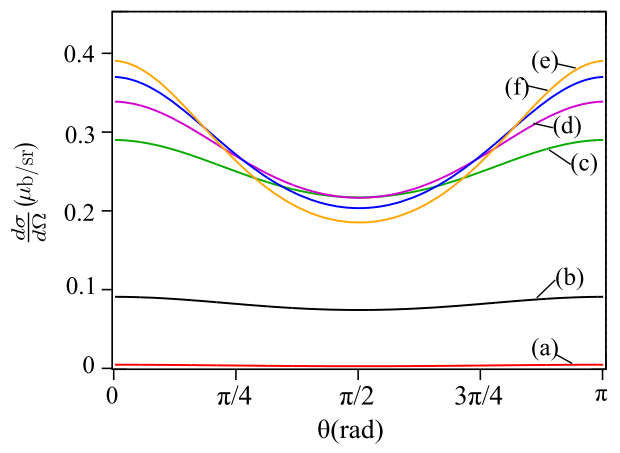

FIG. 4. Differential cross section of $\gamma \gamma$ scattering vs angle for unpolarized photons at various values of the center of mass energy: (a) $\sqrt{s}=0.75 \mathrm{MeV}$, (b) $\sqrt{s}=1 \mathrm{MeV}$, (c) $\sqrt{s}=1.25 \mathrm{MeV}$, (d) $\sqrt{s}=1.5 \mathrm{MeV}$, (e) $\sqrt{s}=1.75 \mathrm{MeV}$, and (f) $\sqrt{s}=2 \mathrm{MeV}$.

two interacting photons have the same energy, the CM frame coincides with the laboratory's. The unpolarized differential cross sections $d \sigma / d \Omega$ for $\gamma \gamma$ collisions, evaluated in the center of mass reference frame for various values of $\sqrt{s}$, are presented in Fig. 4. The angular distributions show that for energies slightly lower or around the peak, the $\gamma \gamma$ differential cross section is quite flat, thus maximizing the emission in the transverse direction.

\section{PRIMARY GAMMA SOURCES}

The primary gamma beams are generated by inverse Compton scattering between a high-brightness electron beam and a high energy laser pulse. The characteristics of the gamma source have been chosen on the basis of the evaluation of the $\gamma \gamma$ luminosity $\mathcal{L}$ around values of the CM energy of about 1-2 MeV. In fact, being the luminosity of the system given by

$$
\mathcal{L}=\frac{f N_{\gamma 1} N_{\gamma 2}}{4 \pi \sigma_{x, \mathrm{IP}} \sigma_{y, \mathrm{IP}}}
$$

with $N_{\gamma 1,2}$ number of gamma photon of the two primary beams, $f$ the repetition rate of the process and $\sigma_{x, y, \mathrm{IP}}$ the transverse dimensions of the primary beams at the interaction point (IP), the event rate turns out to be

$$
N=\sigma \mathcal{L}
$$

Achieving a value of 1 event/hour would require, with photons of energy around $1-2 \mathrm{MeV}(\sigma \approx 2 \mu \mathrm{b})$, a luminosity of $\mathcal{L} \approx 1.3 \times 10^{26} \mathrm{~cm}^{-2} \mathrm{~s}^{-1}$, leading, with $f=100 \mathrm{~Hz}$, to figures as $N_{\gamma 1,2} \approx 1.5 \times 10^{9}$ and $\sigma_{x, y, \mathrm{IP}} \approx 3.7 \mu \mathrm{m}$ per single shot. Such a high number of primary photons can be obtained with a Compton source provided that the electron and photon beams are focused within a radius of a few microns, and is given by the following equation [28]:

$$
N_{\gamma}=\sigma_{T} \frac{N_{e} E_{L}}{h \nu_{L} 2 \pi\left(\sigma_{e}^{2}+\frac{w^{2}}{4}\right)} .
$$

This expression assumes that the electron bunch length is smaller than the transverse beta functions in order to ignore hourglass effects. In our case, due to the strong electron focusing regime, this last condition is not strictly fulfilled and so Eq. (6) constitutes an overestimation of the gamma production. With about $N_{e}=1.5 \times 10^{9}$ electrons focused at about $3.5 \mu \mathrm{m}$ and $0.5 \times 10^{19}$ laser photons focused at $w=12 \mu \mathrm{m}$, and being the Thomson cross section $\sigma_{T}=0.67 \mathrm{~b}$, we obtain just about $1.5 \times 10^{9}$ primary photons. Moreover, the divergence of the gamma rays, besides the natural dependence on $1 / \gamma$, depends also on the emittance of the electron beam as

$$
\sigma_{r, \mathrm{IP}}^{2} \approx \sigma_{e}^{2}+\left[1+\frac{\varepsilon_{n}^{2}}{\sigma_{e}^{2}}\right] \frac{L^{2}}{\gamma^{2}}
$$

$L$ being the distance between the Compton and the $\gamma \gamma$ interaction points.

Collecting the previous formulas, we can therefore express the event number as

$$
N=f \frac{\sigma_{T}^{2} \sigma}{16 \pi^{3} h^{2} e^{2}} \frac{Q^{2} E_{L}^{2}}{\nu_{L}^{2}\left[\sigma_{e}^{2}+\left(1+\frac{\varepsilon_{n}^{2}}{\sigma_{e}^{2}}\right) \frac{L^{2}}{\gamma^{2}}\right]\left(\sigma_{e}^{2}+\frac{w^{2}}{4}\right)^{2}}
$$

showing that $\varepsilon_{n}$ must be quite smaller than $\sigma_{e}$ to obtain a high luminosity. Supposing $\varepsilon_{n} \ll \sigma_{e}^{2} \approx 3.5 \mathrm{mmmrad}$, and the other parameter values as given above, $L$ turns out to be of the order of $2 \mathrm{~mm}$. In this limit, and assuming a perfect matching between electrons and laser $\sigma_{e} \approx \frac{w}{2}$, Eq. (8) can be approximated with $N=0.43 f Q^{2}(\mathrm{nC}) E_{L}^{2}(\mathrm{~J}) \lambda_{L}(\mu \mathrm{m}) / \sigma_{e}^{6}(\mu \mathrm{m})$.

A plot of the expected number of single shot QED events as a function of electron beam spot size and $L$ is shown in Fig. 5 for $\varepsilon_{n}=1.5 \mathrm{~mm} \mathrm{mrad,} \mathrm{given} \mathrm{by} \mathrm{averaging} \mathrm{the}$ emittance between the values on the two planes at the focus. The star represents a possible working point at 1 event/hour.

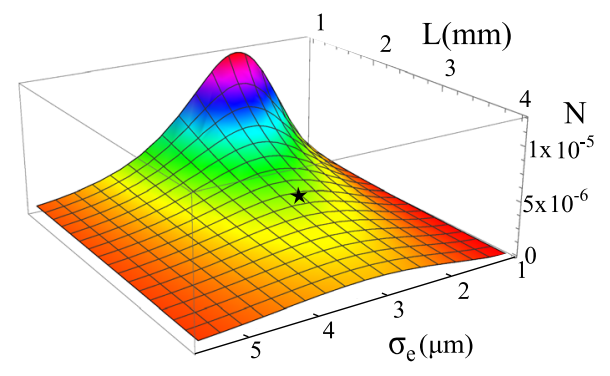

FIG. 5. Single shot number of events $N$ as function of the rms transverse dimension of the electrons and of the distance $L$ between Compton and $\gamma \gamma$ IP. 
TABLE I. Parameters of the Compton sources.

\begin{tabular}{lc}
\hline \hline$\sqrt{s}$ & $\div 2.4 \mathrm{eV}, 2 \mathrm{MeV}$ \\
Electron energy $E_{e}$ & $250 \mathrm{MeV}$ \\
Normalized emittance $\varepsilon_{n, x / y}$ end linac & $0.36 \mathrm{~mm} \mathrm{mrad}$ \\
Normalized emittance $\varepsilon_{n, x / y}$ in the focus & $0.8-2.1 \mathrm{~mm} \mathrm{mrad}$ \\
Electron energy spread $\Delta E_{e} / E_{e}$ & $0.7 \times 10^{-4}$ \\
Charge $Q$ & $250 \mathrm{pC}$ \\
Transverse electron width $\sigma_{e}$ & $1-4 \mu \mathrm{m}$ \\
Laser wavelength $\lambda$ & $1 \mu \mathrm{m}$ \\
Laser waist $w$ & $10 \mu \mathrm{m}$ \\
Laser length & $1 \mathrm{ps}$ \\
Laser energy $E_{L}$ & $1 \mathrm{~J}$ \\
Photon energy $E_{1,2}$ & $<1 \mathrm{MeV}$ \\
Transverse photon beam dimension $\sigma_{r}$ & $1-4 \mu \mathrm{m}$ \\
Repetition rate $f$ & $100 \mathrm{~Hz}$ \\
\hline
\end{tabular}

The parameters of the two Compton sources, well within the state of the art, are listed in Table I.

\section{A. Electron beam line}

The electron beam line has been conceived with the specific aim of preparing an electron beam focused at few microns $(\lesssim 4 \mu \mathrm{m})$ and with a good focal depth. We used therefore a 5.5 cells, standing wave (SW) [SLAC-PUB15107], $\mathrm{x}$-band photoinjector, with accelerating gradient of $200 \mathrm{MV} / \mathrm{m}$ and three $\mathrm{x}$-band traveling wave (TW) cavities $1 \mathrm{~m}$ long, able to achieve $85 \mathrm{MV} / \mathrm{m}$. The $\mathrm{x}$-band technology provides lower emittances and permits more compact devices. Simulations performed with the code ASTRA [22], which takes into account space charge effects, essential in the treatment of the dynamics in the photocathode and in cases of strong focusing, give an emittance of $0.36 \mathrm{~mm}$ $\mathrm{mrad}$ at the end of the linac [21]. The final energy value of $250 \mathrm{MeV}$ was moreover reached in only $5 \mathrm{~m}$ (see Fig. 6). The optimization of the system was done with the genetic code GIOTTO [29], exploiting the efficiency of these algorithms when variables are strongly correlated in a nonlinear way. The beam at the cathode had total charge $Q=250 \mathrm{pC}$, duration $3.15 \mathrm{ps}$ and rms transverse size of $135 \mu \mathrm{m}$. At the exit of the last cavity, the rms longitudinal dimension of the beam was $\sigma_{z} \approx 400 \mu \mathrm{m}$ and the transverse rms dimensions $\sigma_{x}=\sigma_{y} \approx 155 \mu \mathrm{m}$. The focusing stage is

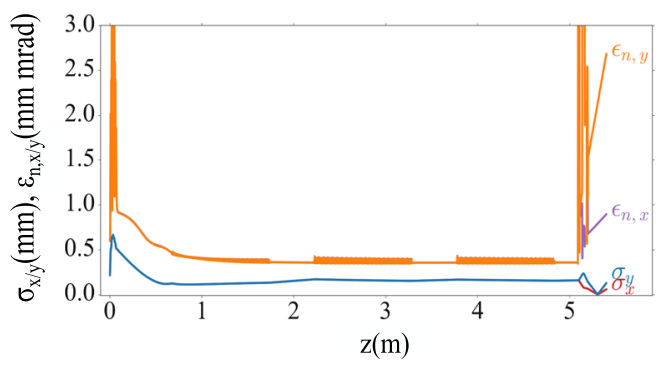

FIG. 6. Electron normalized emittances $\varepsilon_{n, x}, \varepsilon_{n, y}$ and rms transverse $\sigma_{x}, \sigma_{y}$ dimensions along the beam line coordinate $z$.

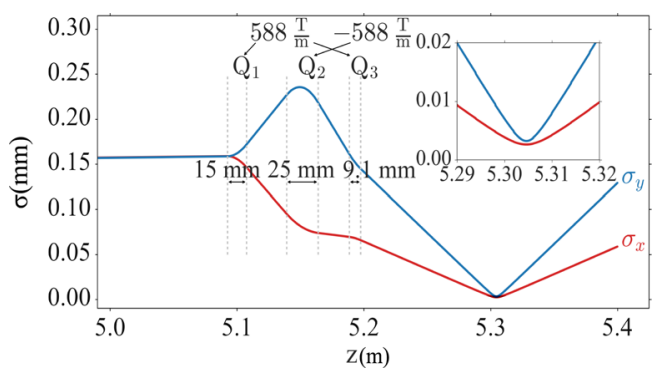

FIG. 7. Focusing stage of the electron beam. Rms transverse $\sigma_{x}$, $\sigma_{y}$ dimensions vs the beam line coordinate $z . Q_{1}, Q_{2}, Q_{3}$ represent the focusing gradients of the quadrupoles. In the inner window, the detail of the focus.

particularly critical. Since the $\gamma$ rays must not impinge on the line elements, the Compton interaction point has to be placed at least at $10 \mathrm{~cm}$ beyond the last focal component. Moreover, the focusing system must guarantee the access of the interaction region to the laser pulse. A triplet of permanent magnet quadrupoles with gradient $g \approx 590 \mathrm{~T} / \mathrm{m}$ and lengths respectively 15.25 and $9.1 \mathrm{~mm}$ was chosen because these elements provide higher gradients with smaller dimensions than the classical ones. In the focus, the rms spot size was $\sigma_{x} \approx 2.6 \mu \mathrm{m}$ and $\sigma_{y} \approx 3.2 \mu \mathrm{m}$, with normalized transverse emittances $\varepsilon_{n, x} \approx 0.79 \mathrm{~mm} \mathrm{mrad}$ and $\varepsilon_{n, y} \approx 2.12 \mathrm{~mm} \mathrm{mrad}$, as shown in Fig. 7. The phase spaces of the electrons before and after the focusing are presented in Fig. 8. The growth of the emittance in the interaction point, which could deteriorate the $\gamma \gamma$ production process, is due to the formation of a halo constituted by relatively few particles. A recalculation of emittance and transverse dimension with a percentage of the total electrons shows that, by eliminating $10 \%$ of the outer particles, the emittances fall down to values close to those at the end of the linac and that the effective radius is considerably smaller, about $1 \mu \mathrm{m}$, as can be appreciated in Fig. 8(d), where the space domain of the focused electrons is shown.
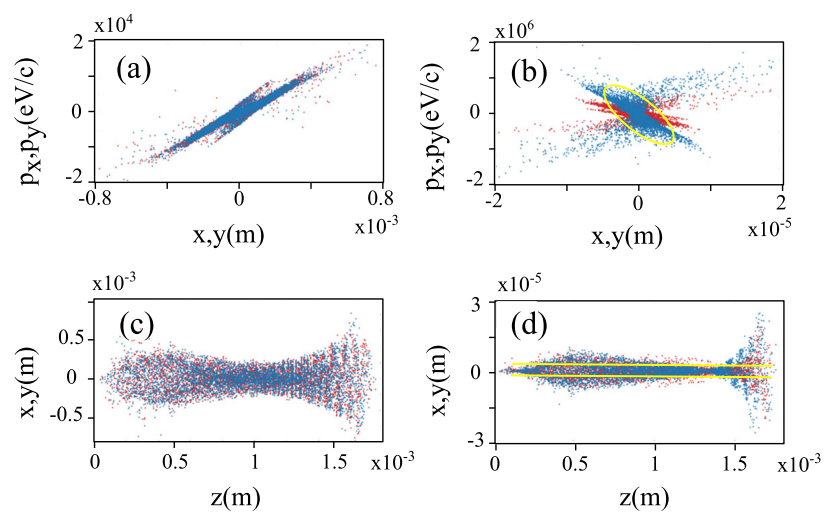

FIG. 8. Electron phase space. (a) and (c) at the end of the linac. (b) and (d) at the Compton interaction point. 


\section{B. Primary gamma beams}

In the Compton interaction the electron beam in the focus collides with an IR laser in the Gaussian mode, whose specifics are listed in Table I. With the adopted laser parameters, the interaction falls in the linear regime, and nonlinear effects are therefore disregarded [30-32]. The conservation of the momentum four-vector for each single Compton interaction leads to the well-known relation between the energy of the radiation $E_{p}$ and the energy of the laser photon $E_{p L}$,

$$
E_{p}=E_{p L} \frac{1-\underline{e}_{k} \cdot \underline{\beta}_{i}}{1-\underline{n} \cdot \underline{\beta}_{i}+\frac{E_{p L}}{m c^{2} \gamma_{i}}\left(1-\underline{e}_{k} \cdot \underline{n}\right)},
$$

where $\underline{\beta}_{i}$ is the velocity of the $i$ th electron, $\underline{e}_{k}$ the unit vector of the laser direction, $\underline{n}$ is the direction of the scattered photon, $h$ the Planck constant and $\gamma_{i}$ the electron Lorentz factor [33]. The Compton radiation polarization properties are described in Ref. [34]. However, for the sake of simplicity, we will assume the photons to be unpolarized. The photon pulse generated by the electron beam described in the previous paragraph in the space domain and after a propagation of $1 \mathrm{~mm}$ is shown in Fig. 9. The transverse rms dimension of the pulse is about $4 \mu \mathrm{m}$.

The natural spectrum of the primary gamma beams is presented in Fig. 10(a), with $1.5 \times 10^{9}$ photons distributed all over the energies from $E_{p L}$ up to $E_{p \text {,max }}$. The maximum energy $E_{p, \text { max }} \approx 4 E_{p L} \gamma_{i}^{2}$ of the primary $\gamma$ rays turns out to be about $1 \mathrm{MeV}$ and the photons spread across a cone of angular dimension $1 / \gamma$. Figure 10(b) is instead relevant to the spectrum after both a slight collimation with acceptance angle $\theta_{\text {acc }}=0.01 \mathrm{rad}$ and a cut in the space domain at one $\sigma$. The number of photons lowers to $9.5 \times 10^{8}$, but the cut concerns only the least energetic part of the spectrum, with $E_{p}<400 \mathrm{keV}$, involved in collisions with low cross section values and reduces almost completely the photon halo, as shown by the shape in cyan in Fig. 9.

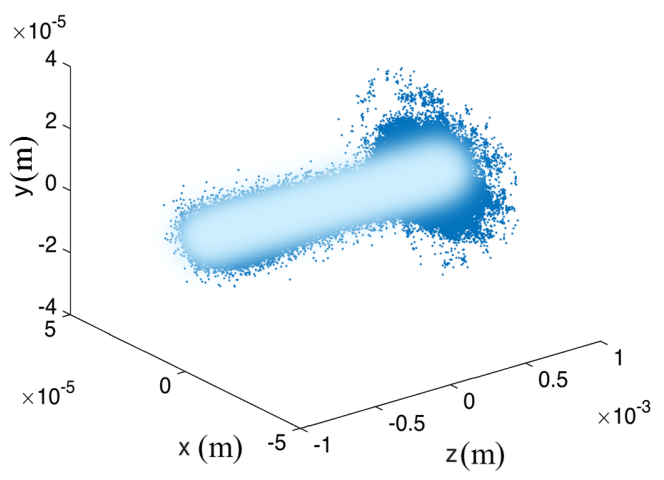

FIG. 9. Compton gamma photons in the space domain. In blue, the whole gamma beam. In light blue, the photon beam after collimation and cut.
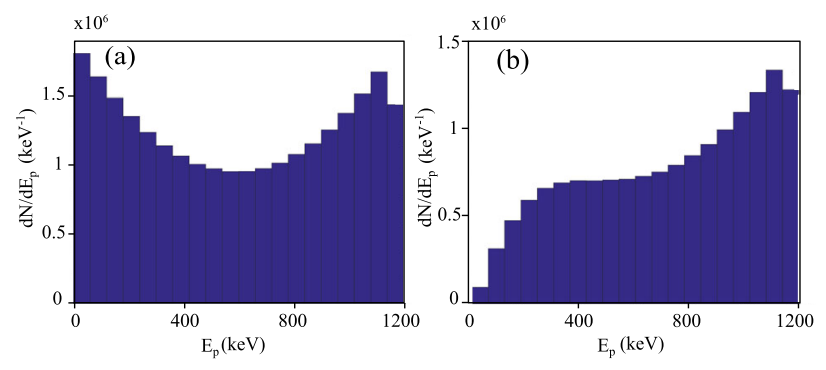

FIG. 10. Compton spectra of (a) natural, (b) collimated at $\theta_{\text {acc }}=0.01 \mathrm{rad}$.

\section{SECONDARY GAMMA BEAM PRODUCTION}

The two symmetric Compton gamma beams collide in an interaction point placed at a distance $L$ from each Compton IP. The interaction region has a transverse extension depending on the distance and on the divergence of the photons, which scales as the inverse of the electron Lorentz factor $\gamma_{e}$. The two primary gamma beams described in the previous section have been divided in cells whose dynamics has been followed in time, as shown in Fig. 11(a). Due to the broad spectrum of the gamma rays, the collisions are in general not symmetric, and a dispersion of the energy in the center of mass $E_{\mathrm{cm}}$ takes place, as shown in Figs. 11(b) and 11(c), where the instantaneous and integrated $E_{\mathrm{cm}}$ distributions are presented. The center of mass Lorentz factor $\gamma_{c}$, in principle, ranges therefore between 1 and $\gamma_{e}$.

However, due to the Compton energy-angle correlation, photons encounter preferably other photons with similar energy, the number of asymmetric collisions is therefore quite contained and the center of mass Lorentz factor $\gamma_{c}$ varies between 1 and 1.5.

The propagation of the gamma beams across the $\gamma \gamma$ interaction point, the spectral width and the asymmetry of the collisions have been taken into account in the evaluation of the number of events of secondary gamma production that has been performed with the new code ROSE (Rate of Scattering Events), developed ad hoc.

Starting from two counterpropagating beams of Compton gamma photons, the code defines a common space grid where the kinematics takes place. The tracking of both beams up to the end of the scattering process permits to dimension the total space window. The time evolution has been discretized over a total of $N_{T}$ steps, starting from the instant where the first collisions take place. For all input couples in each cell, once that the output angles in the center of mass reference frame are randomly sampled, energies and momenta of the generated pair are evaluated, followed by a Monte Carlo procedure for accepting or rejecting the event on the basis of the value of the differential cross section $d \sigma / d \Omega$. Typical parameters are number of primary gamma macroparticles 30000 , total number of cells $100^{3}, N_{T}=50$. 
(1)

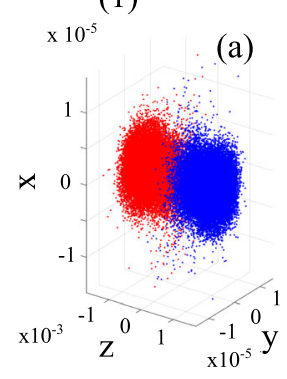

(4)

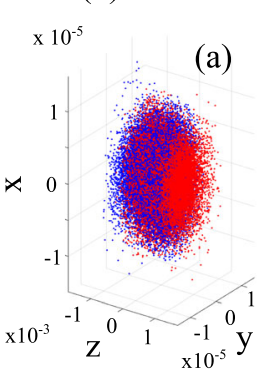

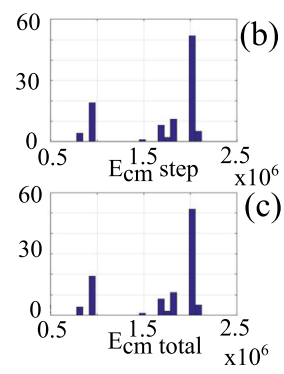

(b)
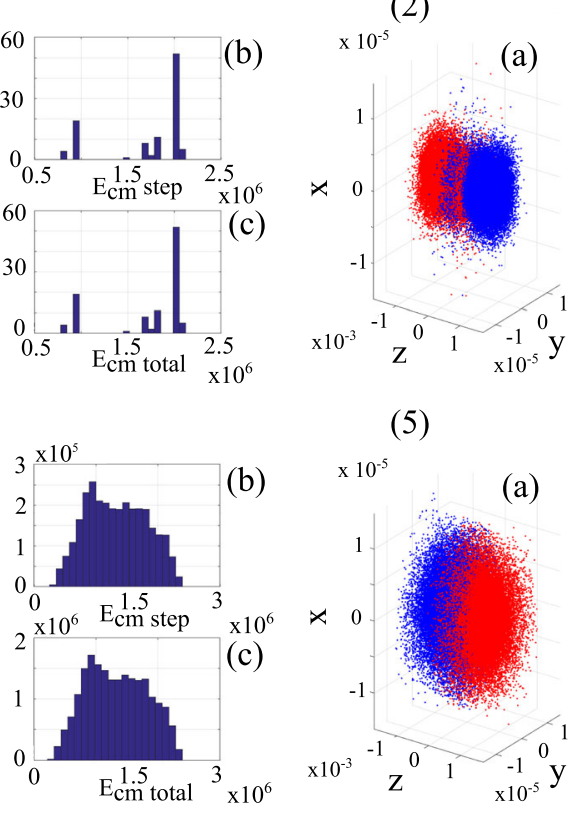

(5)

(2)

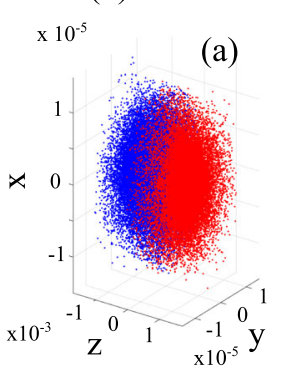

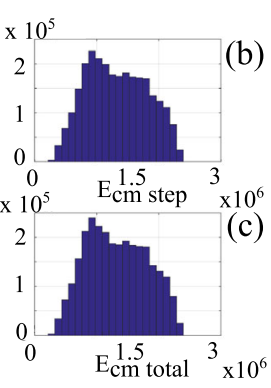

(b)

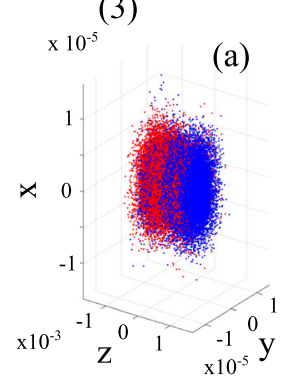

(a)

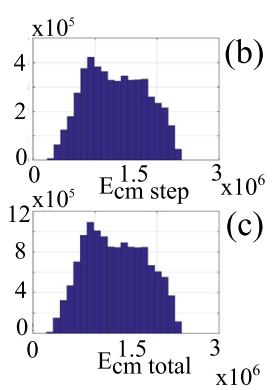

(6)
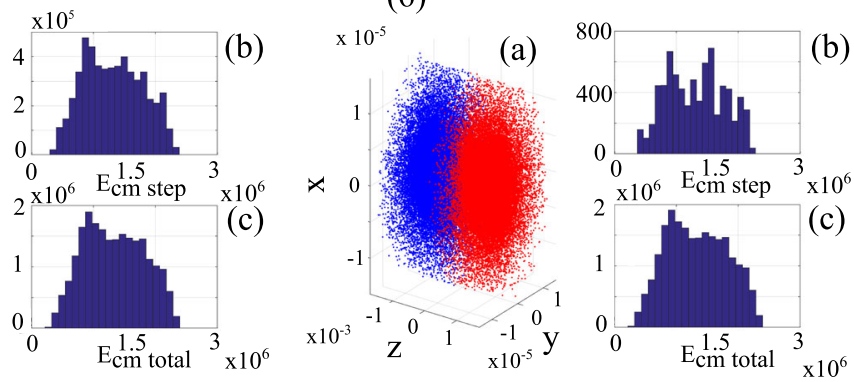

(b)

(c)

FIG. 11. Superposition of the beams in space at various instants (a) and instantaneous (b) and time averaged (c) center of mass energy dispersion. (1) $t=0$ ps. (2) $t=0.2$ ps. (3) $t=0.4$ ps. (4) $t=0.6$ ps. (5) $t=0.8$ ps. (6) $t=1$ ps.

The return to the laboratory system is ruled by the inverse Lorentz transformations. The phase space of the generated pairs provides the angular and energy distributions of the offspring. The system has a substantial azimuthal symmetry. The distribution of the events vs the zenith angle $\theta$ and the energy $E$ in the laboratory frame is presented in Fig. 12.

The most energetic particles are ejected along the axis of the system, but a considerable amount of photon moves perpendicularly. Collecting all data, the total amount of QED events evaluated per single shot and as a function of the distance $L$ between the Compton and $\gamma \gamma$ interaction points is shown in Fig. 13, which presents a trend similar to the one predicted in formula (8). With a repetition rate of

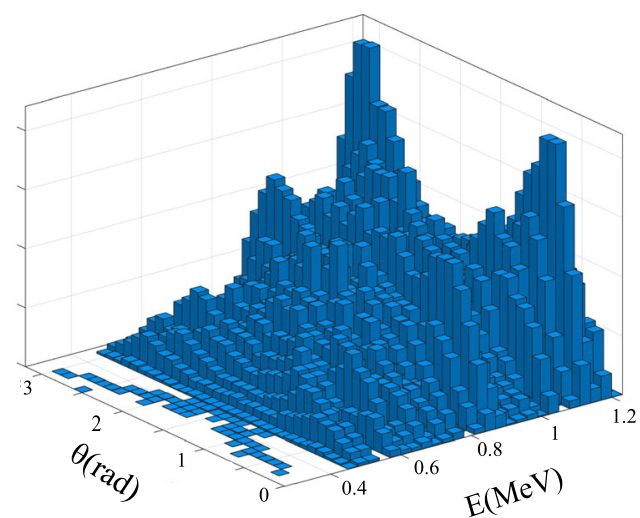

FIG. 12. Distribution of the $\gamma \gamma$ events in the laboratory as a function of the energy of the secondary particles $E=E_{3,4}$ and of the zenith angle $\theta$.
$100 \mathrm{~Hz}$, one event per hour is achieved for $L$ between 1 and $1.5 \mathrm{~mm}$. Tripling the distance $(L=4 \mathrm{~mm})$ would mean passing to a generation rate lower than 0.1 event per hour. The optimum work interval is therefore quite below this value.

\section{BACKGROUND AND DETECTION CONSIDERATIONS}

The gamma-gamma production is not the only process that takes place. Other concomitant processes occur: the Breit-Wheeler pair production $\left(\gamma \gamma \rightarrow e^{+} e^{-}\right)$[24], the triplet pair production (TPP) $\left(\gamma e^{-} \rightarrow e^{+} e^{-} e^{-}\right)$[35], this last occurring as a consequence of the collisions between the primary electrons and gammas, the Møller scattering $\left(\phi e^{-} e^{-} \rightarrow e^{-} e^{-}\right)$between the primary electrons. As regards the Breit-Wheeler scattering, this process is one

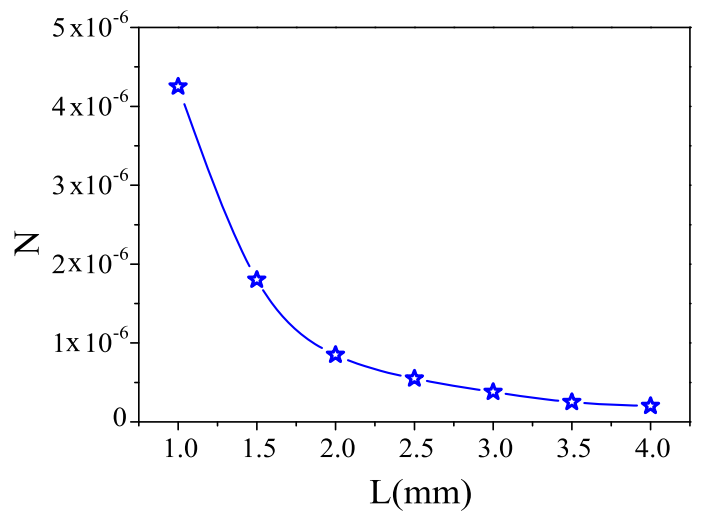

FIG. 13. $\quad \gamma \gamma$ event rate. 


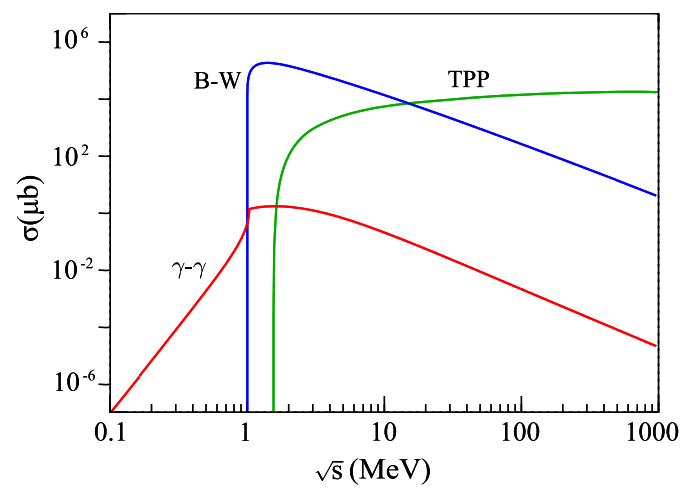

FIG. 14. Comparison between $\gamma \gamma$, Breit-Wheeler and TPP cross sections.

of the manifestations of the mass-energy equivalence and it has never been detected in accelerator experiments due to the difficulty in preparing colliding gamma ray beams, although the more complex multiphoton Breit-Wheeler has been first observed almost twenty years ago [36]. In Fig. 14 the cross sections of $\gamma \gamma$, Breit-Wheeler and TPP scattering events are compared, showing the strong preponderance of the probability of charged particle production with respect to the emission of photons. Strategies for avoiding or containing the background flux on the detector could involve the use of electric or magnetic fields for deflecting the secondary charged particles beams or, in the case TPP and Møller scattering, the development of a geometry which foresees that the primary beams do not encounter each other. This can be achieved by driving the electron beams along slightly curved paths, balancing the advantage of diminishing the background from TPP and Møller scattering, with the decrease of flux due to side Compton interaction.

\section{CONCLUSIONS}

The proposed $\gamma \gamma$ collider is feasible with the present technology and opens the way to new important tests of QED. The setup chosen in this study is based on a conventional linac and a infrared laser, as those of the ELI_NP project. Other devices could be considered, as for instance a $\mathrm{CO}_{2}$ laser. In the scenario described here, furthermore, the initial polarizations can be fully controlled and therefore useful information on the polarizationdependent scattering amplitudes can be extracted. The present evaluations of the event rate and of their energyangle distribution are based not only on scaling laws, but also on a robust set of start-to-end simulations from the electron production stage up to the $\gamma \gamma$ propagation. The Compton backscattering production of high-energy photons poses important challenges in the experimental setup, and the most critical aspect appears to be the focal stage of the electron beam, because the focusing system is the closest material element to the gamma IP and must satisfy several geometrical constraints. Other quite demanding requirements, such as the low electron emittances and energy spread, and the necessity of $100 \mathrm{~Hz}$ repetition rate, the high brilliance of the laser system, have been extensively studied, tested and fulfilled in previous beam dynamics experiments and projects. Beyond the pairproduction threshold several QED processes open up and the observation of gammas from gamma-gamma scattering in this regime requires nontrivial background rejection schemes. Eventually this can lead to strategies for separating the charged particles from photons based on electric or magnetic fields, or the development of geometries avoiding the direct collision between the electron beams and primary gammas, thus decreasing the triplet pair rate production. Also the detection system should be designed ad hoc, starting from the numerous studies already present in the literature. Conversely, the setup can also be considered as a tool for the direct test of the Breit-Wheeler process or as a source of pairs. An accurate treatment of the background processes is beyond the reach of the present paper and will be the object of future work.

[1] O. Halpern, Scattering processes produced by electrons in negative energy states, Phys. Rev. 44, 855 (1933).

[2] P. L. Csonka, Are photon-photon scattering experiments feasible?, Phys. Lett. 24B, 625 (1967).

[3] P. L. Csonka and K. S. Koelbig, Photon-photon scattering with synchrotron radiation, Phys. Rev. D 10, 251 (1974).

[4] P. P. Kane and G. Basavaraju, Possibilities of observation of nonlinear quantum electrodynamic effects in vacuum, Rev. Mod. Phys. 39, 52 (1967).

[5] For a recent review and a collection of references, see G. V. Dunne, Heisenberg-Euler effective Lagrangians: Basics and extensions, in From Fields to Strings: Circumnavigating Theoretical Physics. Ian Kogan Memorial Collection (World Scientific, Singapore, 2005).

[6] J. J. Klein and B. P. Nigam, Birefringence of the vacuum, Phys. Rev. 135, B1279 (1964).

[7] S. L. Adler, Photon splitting and photon dispersion in a strong magnetic field, Ann. Phys. (N.Y.) 67, 599 (1971).

[8] E. Iacopini and E. Zavattini, Experimental method to detect the vacuum birefringence induced by a magnetic field, Phys. Lett. 85B, 151 (1979).

[9] E. Iacopini, B. Smith, G. Stefanini, and E. Zavattini, On a sensitive ellipsometer to detect the vacuum polarization induced by a magnetic field, Nuovo Cimento Soc. Ital. Fis. 61B, 21 (1981).

[10] F. Moulin, D. Bernard, and F. Amiranoff, Photon-photon elastic scattering in the visible domain, Z. Phys. C 72, 607 (1996).

[11] M. Marklund and J. Lundin, Quantum vacuum experiments using high intensity lasers, Eur. Phys. J. D 55, 319 (2009).

[12] F. Della Valle, A. Ejlli, U. Gastaldi, G. Messineo, E. Milotti, R. Pengo, G. Ruoso, and G. Zavattini, The PVLAS experiment: measuring vacuum magnetic birefringence 
and dichroism with a birefringent Fabry-Perot cavity, Eur. Phys. J. C 76, 24 (2016).

[13] A. Cadène, P. Berceau, M. Fouché, R. Battesti, and C. Rizzo, Vacuum magnetic linear birefringence using pulsed fields: status of the BMV experiment, Eur. Phys. J. D 68, 16 (2014).

[14] W. Becker, J. K. McIver, and R. R. Schlicher, Scattering of light by light: Possible experimental detection, Phys. Rev. A 38, 4891 (1988).

[15] W. Becker, J. K. McIver, and R. R. Schlicher, Testing the photon-photon sector of quantum electrodynamics with free-electron lasers, J. Opt. Soc. Am. B 6, 1083 (1989).

[16] K. Homma, K. Matsuura, and K. Nakajima, Testing helicity-dependent $\gamma \gamma \rightarrow \gamma \gamma$ scattering in the region of MeV, Prog. Theor. Exp. Phys. (2016) 013C01.

[17] E. L. Saldin, E. A. Schneidmiller, and M. V. Yurkov, FEL system for gamma-gamma collider at TESLA, Nucl. Instrum. Methods Phys. Res., Sect. A 472, 94 (2001).

[18] P. Chen, T. Ohgaki, A. Spitkovsky, T. Takahashi, and K. Yokoya, Simulations of the interaction region in a photonphoton collider, Nucl. Instrum. Methods Phys. Res., Sect. A 397, 458 (1997).

[19] E. Milotti et al., Exploring quantum vacuum with lowenergy photons, Int. J. Quantum. Inform. 10, 1241002 (2012).

[20] A. Torre, G. Dattoli, I. Spassovsky, V. Surrenti, M. Ferrario, and E. Milotti, Double free-electron laser oscillator for photon-photon collisions, J. Opt. Soc. Am. B 30, 2906 (2013).

[21] A. Bacci et al., Electron Linac design to drive bright Compton back-scattering gamma-ray sources, J. Appl. Phys. 113, 194508 (2013).

[22] K. Floetmann, AstRA, http://desy.de/mpyo/Astra_ dokumentation/.

[23] K. Yokoya, http://www-acc-theory.kek.jp/members/cainS/.
[24] G. Breit and J. A. Wheeler, Collision of two light quanta, Phys. Rev. 46, 1087 (1934).

[25] B. De Tollis, Dispersive approach to photon-photon scattering, Nuovo Cimento 32, 757 (1964).

[26] B. De Tollis, The scattering of photons by photons, Nuovo Cimento 35, 1182 (1965).

[27] V. Costantini, B. De Tollis, and G. Pistoni, Nonlinear effects in quantum electrodynamics, Nuovo Cimento A 2 , 733 (1971).

[28] G. A. Krafft and G. Priebe, Compton sources of electromagnetic radiation, Rev. Accel. Sci. Techol. 03, 147 (2010).

[29] A. Bacci, C. Maroli, V. Petrillo, A. Rossi, and L. Serafini, Maximizing the brightness of an electron beam by means of a genetic algorithm, Nucl. Instrum. Methods Phys. Res., Sect. B 263, 488 (2007).

[30] F. Albert et al., Characterization and applications of a tunable, laser-based, $\mathrm{MeV}$-class Compton-scattering $\gamma$-ray source, Phys. Rev. ST Accel. Beams 13, 070704 (2010).

[31] D. Seipt and B. Kampfer, Scaling law for the photon spectral density in the nonlinear Thomson-Compton scattering, Phys. Rev. ST Accel. Beams 14, 040704 (2011).

[32] C. Maroli, V. Petrillo, P. Tomassini, and L. Serafini, Nonlinear effects in Thomson backscattering, Phys. Rev. ST Accel. Beams 16, 030706 (2013).

[33] V. Petrillo et al., Photon flux and spectrum of $\gamma$-rays Compton sources, Nucl. Instrum. Methods Phys. Res., Sect. A 693, 109 (2012).

[34] V. Petrillo et al., Polarization of $\mathrm{x}$-gamma radiation produced by a Thomson and Compton inverse scattering, Phys. Rev. ST Accel. Beams 18, 110701 (2015).

[35] V. Votruba, Pair production by $\gamma$-rays in the field of an electron, Phys. Rev. 73, 1468 (1948).

[36] C. Bamber et al., Studies of nonlinear QED in collisions of 46.6 GeV electrons with intense laser pulses, Phys. Rev. D 60, 092004 (1999). 